\title{
EL PROYECTO ARTÍSTICO COMO RELATO EN EL ARTE CONTEMPORÁNEO: CASOS, DISCUSIÓN Y CONCLUSIONES
}

\author{
José Vicente Martín Martínez
}

Universidad Miguel Hernández de Elche

\section{Resumen}

El artículo analiza el papel del relato en el arte contemporáneo, entendido como horizonte de sentido, y cómo se ha convertido en la base de las prácticas basadas en el proyecto. A través tanto de la comparación con conceptos propios de la teoría literaria -historia, discurso e hipertexto- como del estudio de diversos casos a modo de ejemplos, se pretende analizar la naturaleza y características de este modelo de producción y recepción artística.

Palabras claves: RELATO; DISCURSO; PROYECTO ARTÍSTICO; HIPERTEXTO; NARRACIÓN

\section{THE ART PROJECT AS STORY IN CONTEMPORARY ART: CASES, DISCUSSION AND CONCLUSIONS}

\section{Abstract}

The article analyzes the role of the story in contemporary art, understood as a horizon of meaning, and how it has become the basis of project-based practices. Through the comparison with concepts borrowed from literary theory such as story, discourse and hypertext as well as the study of various cases as examples, the aim is to analyze the nature and characteristics of this model of art production and reception.

Keywords: STORY; DISCOURSE; ARTISTIC PROJECT; HYPERTEXT; NARRATION

\footnotetext{
Martín Martínez, José Vicente. "El proyecto artístico como relato en

el arte contemporáneo: Casos, discusión y conclusiones". AusArt 6 (1): 195-203. D0I: 10.1387/ausart.19526
}

\section{AUSART}


1. Important artifacts and personal property from the collection of Lenore Doolan and Harold Morris, including books, street fashion, and jewelryes, es una singular obra de Leanne Shapton (2001). Se trata de una historia de amor escondida en la apariencia de un catálogo de subastas. Los objetos de la relación entre Lenore y Harold son supuestamente subastados por lotes y, de este modo, podemos recorrer la vivencia de su relación a través de los enseres personales que fueron testigos de ella y las fotografías que las atestiguaron. Así presentada, la historia no tiene un principio y un final, pues los lotes no se organizan de un modo cronológico, provocando que el espectador acceda a la historia con la aleatoriedad de quien lee un catálogo comercial.

2. Building Stories es una novela gráfica de Chris Ware (2012) que se presenta en una caja de grandes dimensiones, $42 \times 30 \times 5 \mathrm{~cm}$, como si de un juego de mesa se tratara. En el interior de la caja se recogen catorce elementos diferentes: libros de tapa dura y rústica, de muy diversos tamaños y formatos, incluyendo algo que podría entenderse como el tablero del juego de mesa al que la caja remite. Las catorce unidades aparecen de modo independiente por lo que el lector puede leerlas en el orden que desee. Componen una visión fragmentaria y caleidoscópica del universo familiar y doméstico de la protagonista femenina a través de las distintas etapas de su vida. Si bien en la versión española se ha optado por la traducción Fabricar historias, el título inglés también podría traducirse como Historias de edificios, lo cual alude a la importancia del hogar en la narración. El lector, embargado por el particular aire de cercanía y extrañamiento propio de las obras del autor americano, es testigo de la monótona vida de la protagonista: sus dudas, deseos y frustraciones.

3. Kutluğ Ataman es un artista de origen turco, algunos de cuyos trabajos tienen la apariencia formal del cine documental. En una de sus obras más significativa, su vídeo instalación Küba (2005), recoge entrevistas a cuarenta personas que habitan en un gueto en Estambul. Las entrevistas se muestran en monitores y mobiliario de estética obsoleta. En total la obra implica treinta dos horas de metraje y cuarenta monitores. Este formato de pantallas múltiples, habitual en la actualidad en galerías y museos, provoca que, en palabras del propio Ataman, las "obras sean imposibles de mirar", para añadir que no conoce a nadie, excepto él, que haya visto la obra completa (Thornton 2015, 96).

A. En su obra El paradigma del arte contemporáneo. Estructuras de una revolución artística, Nathalie Heinich (2014) analiza las características propias del cambio de paradigma que significa el arte contemporáneo frente al arte 
moderno. En primer lugar, conlleva la ampliación de los límites, los materiales y los usos tradicionales de la obra de arte, buscando ampliar el territorio de lo que es susceptible de ser considerado como arte. La segunda transformación enlaza con la idea de la inserción de la obra en un contexto de recepción específico, tanto físico como socio-cultural. La tercera transformación, que es la que aquí nos interesa, alude a la necesidad de construir un relato que transcienda la materialidad de la obra y la inscriba en un horizonte de sentido más complejo. En palabras de Heinich (2017, 96): "El relato: este es el punto en común de estas múltiples formas de proyectar la obra más allá del objeto. (...) Puede decirse que el arte contemporáneo se ha convertido, esencialmente, en un arte de "contar": un arte de la narración, o incluso de la leyenda, un arte del comentario, de la interpretación (...) El objeto es sólo un pretexto, como mucho un activador, que inducirá acciones, palabras, operaciones, reconfiguraciones del espacio (...) y la obra está en este conjunto de cosas".

Nótese que en 1, 2, y 3 la presencia de una imagen ilustrativa de las obras es, hasta cierto punto, prescindible, es decir, no es estrictamente necesaria para que podamos imaginarnos sus universos narrativos o de sentido. En esta misma argumentación se inscriben las palabras del propio Kutlug Ataman: "mis obras, en realidad, no son productos, son papeles que se escriben al finalizar un esfuerzo de pensamiento" (citado en Thornton 2015, 95). Frente al producto, frente al objeto desfuncionalizado y estetizante del arte moderno, el arte contemporáneo se presenta como proceso que es narrado, constituyendo este relato el horizonte de sentido que conforma las prácticas artísticas del nuevo paradigma. Así, la obra de arte contemporáneo transciende el objeto, configurándose como proyecto, esto es, como una potencialidad nunca definitivamente cerrada, que se edifica desde un relato que la dota de sentido.

4. Taryn Simon es una artista multidisciplinar americana que trabaja desarrollando una idea -o relato- a través de soportes como la fotografía, el texto o la escultura. Su obra An American index of the hidden and unfamiliar (2007), se expone como un conjunto de fotografías tradicionales que, sin embargo, aparecen junto a textos explicativos en la publicación/catálogo. El proyecto recoge un inventario de objetos, lugares y personas que muestran aspectos de la cultura norteamericana relacionados con la ciencia, la seguridad, la religión, el entretenimiento o la naturaleza que normalmente permanecen ocultos al espectador habitual: un almacén de residuos nucleares, un depósito de alimentos requisados en la aduana del aeropuerto John F. Kennedy de Nueva York, una unidad de preservación criogénica, un quirófano donde se practica una reconstrucción de himen... En otra de sus obras, The Innocents (2002), 
Simon documenta la historia de individuos que cumplieron condena por crímenes que no cometieron, retratándolos en lugares relacionados con el caso: el lugar de la coartada que no fue considerada, la escena del crimen, la escena del arresto, el lugar donde un informante creyó oír la confesión del acusado.... Desvelando lo oculto y haciéndonos partícipe del privilegio de su acceso, su trabajo adopta estrategias propias del periodismo de investigación que tiene como aspiración desvelar la verdad a los espectadores/lectores.

a. Este papel del relato en el arte contemporáneo puede ponerse en relación con la distinción propia de la teoría literaria entre historia y discurso como elementos de la narración (Chatman [1978] 2013). La historia se refiere a la totalidad de la narración, a todos los posibles personajes, lugares, sucesos que la componen y a la línea de tiempo en que se ubican. El discurso alude a cómo se presentan una selección de los acontecimientos en una materialización específica: el medio, el énfasis en unos hechos $u$ otros, en unos personajes u otros, el orden en que se presentan los eventos. Frente al discurso entendido como el cómo, la historia no sería sólo el qué, si no que se constituiría en términos de un universo ficcional que prevé y contiene cualquier concreción material, cualquier soporte, cualquier selección de acontecimientos, cualquier énfasis, cualquier discurso.

Este carácter potencialmente polimorfo de los diversos discursos que provienen de una única historia se pone en evidencia en lo que, en la cultura audiovisual contemporánea, se ha dado en llamar transmedia (Giovagnoli 2011, 26), esto es, aquellas narraciones que se materializan en muy diversos medios y soportes, estableciendo un entramado de relaciones que aluden a una única historia, como podemos ver en las sagas de ciencia ficción como Star Wars o Star Trek, que generan precuelas y secuelas, narraciones en cine, cómic, novela, etc... que aluden a un universo ficcional en expansión, pero cuya potencialidad preexiste. Es decir, la historia se supone como anterior e hipotéticamente definida y prevé o anticipa cada uno de los sucesos, escenarios y actores que aparecen en los diferentes discursos (Chatman [1978] 2013, 49).

5. Este aspecto anticipador y abstracto de la historia frente a la inmediatez y materialidad del discurso, podría ser ilustrado por la anécdota contada por Jorge Luis Borges en la entrevista del programa de Televisión Española "A fondo" de 1976. En el contexto de la relación con su madre, el escritor argentino relata su dificultad para encontrar la manera de finalizar un cuento, "La intrusa”, y cómo recibió su ayuda. El cuento narra la relación entre dos hermanos que se ve alterada por una mujer de la cual ambos se enamoran. El mayor 
de ellos decide matarla para evitar que se interponga entre ellos y Borges explica en la entrevista que no sabía cómo podría comunicarle el hermano mayor al pequeño lo que había hecho porque "toda la suerte del cuento dependía de eso, dependía de las palabras". Borges le hace saber a su madre sus dudas y el escritor argentino nos cuenta:

"Entonces madre me dijo «démaje pensar»(...) Y luego me dijo con la voz distinta, "ya sé lo que le dijo», como si hubiera ocurrido eso, como si no hubiera sido un sueño mío, esa historia. "Ya sé lo que le dijo»(...) «A trabajar, hermano, esta mañana la maté». Empieza recordándole al otro que son hermanos y le ordena que trabaje con él para enterrar el cadáver y luego después viene la aclaración (...) Todo eso lo intuyó mi madre, yo no hubiera dado con una frase tan feliz. Además, eso: "ya sé lo que le dijo», es decir que en ese momento ella creía en mis mundos imaginarios. Ella se había identificado conmigo, de tal modo, mejor todavía, porque yo no hubiera encontrado la frase. Ella los conocía mejor que yo, que los había imaginado. Es extraordinario ese momento".

La anécdota ilustra de modo elocuente este carácter previo y potencial de la historia, su entidad totalizadora, pero también el hecho de constituirse como territorio potencial por descubrir siempre que se sea capaz de comprender su lógica narrativa y/o de sentido.

6. En una entrevista con motivo de su intervención en el Minneapolis Institute of Art en 2013, Mark Dion define su trabajo del siguiente modo: "Algunas personas pintan, algunas personas toman fotografías, algunas personas esculpen... Yo compro, ése es mi medio como artista, eso es lo que hago, compro cosas y las reorganizo o reorganizo el material que pertenece a instituciones, museos y otras cosas. Todo mi trabajo está investido en la importancia de la cultura material y la creencia de que los objetos hablan". Mark Dion es conocido por sus acumulaciones de objetos dispuestos a modo de gabinete de curiosidades o cámara de maravillas, replicando las estructuras culturales de presentación, ordenación y sistematización de la verdad como hecho cultural. $A$ veces sus instalaciones son reordenaciones de los fondos de colecciones de museos etnográficos o antropológicos como su intervención en el Museo de la Universidad de Minnesota, Cabinet of curiosities (2001). En otras ocasiones, son acumulaciones de objetos ordenados en torno a un concepto cultural como en su proyecto Concerning hunting (2009), una acumulación de objetos relacionados con la caza: trampas y armas, equipamiento de cazador, animales disecados, incluso puestos de caza reconstruidos. O también proyectos de 
catalogación de la historia cultural como el proyecto Tate Thames Dig (1999), donde Dion, junto a un grupo de voluntarios, extrae del lecho del río Támesis objetos que son expuestos ordenados de modo sistemático.

B / b. Esta distinción entre historia y discurso puede ser trasladada desde la teoría literaria al ámbito de la creación artística, estableciendo un paralelismo entre la historia, entendida como el marco que precede a los discursos y que potencialmente los abarca, con la idea de relato -o proyecto- artístico. El relato-proyecto se entendería de este modo como concepto, propuesta de sentido, historia, que necesita ser concretada en un discurso artístico cada vez más interdisciplinar, cada vez más compuesto por ramificaciones, cada vez más polimorfo, más interactivo, más complejo.

c. Frente a la narración tradicional en la que los acontecimientos se desarrollan en un flujo temporal que, si bien puede ser alterado por analepsis y prolepsis, fluye de modo unidireccional, podemos encontrar modelos narrativos que rompen esa linealidad (Chatman 2013). Por una parte, tendríamos las historias multiformes en las que una única situación o argumento se presenta en múltiples versiones, puntos de vista diferentes que serían excluyentes en nuestra experiencia habitual, cuestionando de este modo la percepción del tiempo y el espacio como verdad absoluta. Superando el formato lineal, se pretende enriquecer la experiencia cotidiana con la posibilidad de realizaciones paralelas múltiples. Acudiendo al cine para ejemplificar este modo narrativo, encontraríamos este tipo de narración en filmes como ;Qué Bello es vivir! (What a wonderful life!, Frank Capra, 1946), Rashomon (Kurosawa, 1950), Atrapado en el tiempo (Groundhog Day, Harold Ramis, 1993), Jackie Brown (Quentin Tarantino, 1997), Existenz (David Cronenberg, 1999) o El efecto mariposa (The Butterfly effect, Erik Bress y Mackye Gruber, 2004). Por otra parte, tendríamos las anti historias, donde los sucesos de la trama no son resueltos, si no que se revela un estado de las cosas. Frente a la trama tradicional, que busca una solución, en las anti historias se propone una exposición de lo narrado, centrada más en los personajes y las situaciones. Encontraríamos ejemplos de este modo de narrar en películas como Short cuts (Robert Altman, 1993), Smoke (Paul Auster y Wayne Wang, 1994), Happiness (Todd Solondz 1998), Magnolia (Paul Thomas Anderson, 1999), Lost in traslation (Sofia Coppola, 2003) o Crash (Paul Haggis, 2004).

Las narraciones no lineales y las anti historias ponen en evidencia la relación entre historia y discurso en la medida en que deconstruyen el discurso tradicional, permitiéndonos de este modo acceder a la esencia de la historia, 
en sus potenciales múltiples puntos de vista, liberada de la dependencia del devenir del suceso y su resolución.

7. Un gran número de los proyectos de Joan Fontcuberta pueden inscribirse dentro del género del falso documental en el que se presentan hechos de ficción con la apariencia formal del discurso institucionalizado de la verdad, científica y/o histórica. Así ocurre en el proyecto Fauna (1988), realizado en colaboración con Pere Formiguera. Fauna es una instalación realizada con material asociado a las exposiciones científicas propias de un museo de historia natural o zoología: minuciosas notas y estudios de campo, fotografías científicas, esqueletos y especímenes disecados... que documentan el hipotético hallazgo del archivo del naturalista alemán Peter Ameisenhaufen -nacido en Múnich en 1895 y desaparecido de forma misteriosa en 1955- y de su ayudante Hans von Kubert en cuyas expediciones descubrieron la supuesta existencia de especies inverosímiles como el Myodorifera Colubercauda, un roedor cuya cola es un reptil; el Alopex Stultux, una especie de tortuga bípeda con su parte exterior en forma de cono y cubierta de pelo que se mimetiza con el entorno hincando su cabeza sobre el terreno y colocándose de modo vertical como si fuese una especie de arbusto o el Centaurus Neandentalensis, un simio cuadrúpedo. El fotógrafo catalán reflexiona de este modo sobre los fundamentos de estas convenciones propias de los procesos de producción y transmisión del conocimiento, cuestionándolos al presentarnos con su formato y apariencia una historia inventada, ciertamente inverosímil.

d. Un paso más allá en la revelación de la dialéctica entre historia y discurso lo podríamos encontramos en el hipertexto como estructura narrativa. Podemos definir el hipertexto como un conjunto de textos y "documentos" (lexias o nodos) no jerarquizados, unidos entre ellos por enlaces que el lector puede activar y que permiten un acceso rápido a cada uno de los elementos constitutivos de ese conjunto (Landow 2006). El hipertexto es por lo tanto un tipo de texto interactivo, no lineal (o multilineal), cuya secuencialidad puede variar considerablemente a lo largo de la lectura en función de las decisiones de cada lector. El hipertexto, como territorio transitable por el lector-espectador, establece una estructura narrativa en la que la lógica lineal se rompe en aras de una mayor participación del lector-espectador. El hipertexto impide una lectura única, promoviendo una abierta e interactiva. Este recorrido del lector por los itinerarios, previstos, ciertamente, por el autor, pero ampliamente abiertos a su elección, nos remite al abandono de un paradigma narrativo basado en lo temporal en beneficio de otro basado en lo espacial. (Clement 2006) Este transitar por la narración hipertextual, puede compararse con el deambular, tanto 
físico como mental, del espectador alrededor de los múltiples registros en los que el relato-proyecto artístico se materializa, eligiendo y asociando aquellos elementos de su interés.

C. A modo de conclusión, podemos afirmar que la obra de arte contemporánea ya no se construye sobre un objeto que remite a su estricta inmanencia, si no sobre un relato que se constituye como horizonte de sentido más allá de una concreción formal especifica. La presencia de este relato se evidencia en aquellas obras que definimos como proyectos, aludiendo a su carácter abierto y a sus múltiples posibilidades de formalización. El relato en la obra de arte contemporáneo tendría así una voluntad integral y totalizadora, provocando un desplazamiento del foco de interés desde la materialidad de los registros formales, hacía la necesaria construcción de este universo ficcional o argumentativo. En la búsqueda de explicitar el sentido de su relato, el proyecto artístico propio del arte contemporáneo tiene una vocación interdisciplinar y multimedial, valiéndose así de cualquier elemento que permita un desvelamiento más apropiado de su sentido.

La comparación con conceptos importados de la teoría literaria, como la oposición historia-discurso, nos puede permitir comprender mejor la relación entre el relato-proyecto y su materialización formal. El papel de la historia en cuanto que constituye el universo ficcional que prevé y contiene cualquier potencial narración, cualquier materialización, cualquier discurso, puede entenderse de modo similar al que tiene el relato-proyecto en el arte contemporáneo, como hemos visto, horizonte de sentido susceptible de materializarse por medio de múltiples y variados soportes.

Del mismo modo, la aplicación del concepto de hipertexto al terreno de la práctica artística contemporánea, puede ayudarnos a revelar la naturaleza de la función que el relato adquiere. En la medida que el hipertexto supone la ruptura de la linealidad del discurso narrativo, la lectura hipertextual se asemejaría al deambular físico y especulativo del lector-espectador por los distintos elementos que componen el discurso multimedial en la búsqueda del desvelamiento de la esencia de su relato-proyecto. Como el escritor hipertextual, el artista que trabaja con la idea de proyecto, debe prever los itinerarios que el lector-espectador puede realizar, y, al dotarle de libertad de elección debe valorar qué parte de los materiales concretos, de los itinerarios no serán transitados por todos los lectores-espectadores. Lo cual implica que la materialización del proyecto debe prever estas "pérdidas" o "descartes" debidas a la falta de atención o interés por parte del espectador, de modo que el sentido no 
se vea alterado. La ausencia de algunos elementos de las obras aquí descritas no implicaría que no pudiera captarse el sentido de su relato, la naturaleza de cada proyecto. En definitiva, el proyecto artístico compartiría con las estrategias hipertextuales el hecho de ofrecer una lectura a la vez fragmentaria y holística en la búsqueda del desvelamiento de su historia-relato.

\section{Referencias}

Ataman, Kutluğ. 2006. "Küba: Journey against the current". Reportaje producido por TBA21. Vídeo de Vimeo, 13:16. Publicado 17 jul. 2012. https://vimeo.com/45905756

Borges, Jorge Luis. 1976. "José Luis Borges" Entrevista por Joaquín Soler Serrano en el programa $A$ fondo. TVE. Vídeo de Youtube. 1:29:52. https://www.youtube.com/watch?v=2gu9l_TqS8I

Chatman, Seymour (1978) 2013. Historia y discurso. La estructura narrativa en la novela y el cine. Traducción de María Jesús Fernández Prieto. Barcelona: RBA

Clement, Jean. 2006. "El hipertexto de ficción, ¿el nacimiento de un nuevo género?". En Teoría del hipertexto: La literatura en la era de la electrónica, $M^{a}$ Teresa Vilariño Picos \& Anxo Abuín González, eds., 13-33. Madrid: Arco Libros

Coles, Alex \& Dion, Mark. 1999. Mark Dion:Archaeology. Text, Renfrew Colin, Williams Robert et al. London: Black Dog

Dion, Mark. 2010. Mark Dion: Concercing hunting. Curated by Dieter Buchhart \& Verena Gamper. Ostfildern-Ruit: Hatje Cantz

- . 2001. Cabinet of curiosities: Mark Dion and the university as installation. Colleen J. Sheehy, editor. Minneapolis MN: University of Minnesota

— . 2013. "MIA artist in residence: Mark Dion". Entrevista, Minneapolis Institute of Art. Vídeo de Youtube. 2:11. https://www.youtube.com/watch?v=LSfn73WCQEs

Fontcuberta, Joan. 2001. Contranatura: Joan Fontcuberta. Alicante: Museo de la Universidad de Alicante

Giovagnoli, Max. 2011. Transmedia storytelling: Imagery, shapes and techniques. Pittsburgh PA: Entertainment Technology Center

Heinich, Nathalie. (2014) 2017. El paradigma del arte contemporáneo: Estructuras de una revolución artística. Traducción de Agustín Temes y Étienne Barr. Madrid: Casimiro

Landow, George P. (2006) 2009. Hipertexto 3.0. Teoría crítica y nuevos medios en la era de la globalización. Traducción de Antonio José Antón Fernández. Barcelona: Paidós

Shapton, Leanne. 2009. Important artifacts and personal property from the collection of Lenore Doolan and Harold Morris, including books, street fashion, and jewelry. New York: Sarah Crichton

Simon, Taryn. 2015. Rear views: A star-forming nebula, and the office of foreign propaganda: The works of Taryn Simon. Londres: Tate

Thornton, Sarah. 2015. 33 artistas en 3 actos. Traducción de Teresa Arijón. Barcelona: Edhasa Ware, Chris. 2012. Building stories. London: Random House 